\title{
IX. Ueber die Zusammensetzung einiger Tellurmineralien.
}

\author{
Von \\ W. Muthmann und E. Schröder in Munchen.
}

Im Anschluss an Untersuchungen uber von uns dargestelite isomorphe Selen- und Tellurverbindungen, deren Ergebnisse bei einer anderen Gelegenheit wiedergegeben werden sollen, hatlen wir uns die Aufgabe gestellt, die Zusammenselzung einiger theils schon mehrmals bestimmter Tellurmineralien zu ermitteln. Nach früheren Arbeiten waren oder konnten dieselben selenhaltig sein; besonders war dies von verschiedenen Forschern in einigen Tetradymiten, jedoch immer nur in Spuren nachgewiesen. Wir waren nun von dem Gedanken geleitet, dieses Selen möglichst quantitativ zu ermitteln und den Einluss desselben auf die chemische Constitution des Minerals zu beobachten, denn es erschien uns von Interesse, die Kenniniss der natürlichen Beispiele, in welchen die Elemente Selen und Tellur in isomorpher Mischung unter sich und mit Schwefel nachgewiesen sind, noch mehr zu erweilern. War nun auch der Erfolg speciell nach dieser Richtung kein positiver, so haben sich aus den nachstehenden Analysen theils Uebereinstimmungen mit dem Ergebniss fruherer Arbeiten, theilweise aber auch neue Resultate folgern lassen. Wir haben zwei Tetradymite (von Orawicza und von Schubkau) und ein von Cumberland stammendes Tellurmineral untersucht, uber welches schon Rammelsberg ${ }^{1}$ Angaben gemacht bat. Das zum Theil sehr schälzenswerthe Material ist der hiesigen mineralogischen Sammlung des Staates eninommen; wir verdanken dasselbe der Liebenswürdigkeil des Herrn Prof. Dr. P. Groth.

Die qualitative Prufung geschah nach den gewohnten Melboden. Zum Nachweise des voraussichtlich doch nur in geringer Menge vorhandenen Selens neben dem Tellur haben wir die verschiedenen bisher bekannten

1) Dessen Mineralchemie 2. Aufl., 1875, S. 5. 
Erfahrungen verwerthet, nach denen theilweise auch Methoden zur quantitativen Trennung vorgeschlagen und in Anwendung sind. Eine sichere Methode bei geringem Selen- und vorherrschendem Tellurgehalt ist jedenfalls die Spectralanalyse ${ }^{1}$ ), wodurch bei einiger Uebung das Selen leicht neben Tellur zu erkennen ist. Divers und Shimosé. ${ }^{2}$ ) beschreiben das Verhalten von Selendioxyd und Tellurdioxyd in concentrirter Schwefelsäure gegen schwellige Säure, wodurch nur Selen gefällt wird, während Tellur erst auf Zusatz von Salzsäure fällt, und grunden hierauf eine Methode zur quantitativen Trennung beider Elemente. Diese Methode ist rasch und leicht auch zum qualitativen Nachweise verwendbar. Erst vor kurzem haben Gooch und Peirce $\left.{ }^{3}\right)$ eine neue Art der quantitativen Trennung vorgeschlagen, die auf der verschiedenen Fluchtigkeit der Bromide basirt. Waren wir auch mit dem Ergebnisse der hiernach angestellten quantitativen Versuche nicht zufrieden, so lässt sich diese Methode zu dem qualilativen Nachweise des Selens sehr wohl verwenden. Unter gewissen Bedingungen, wenn nämlich der Selengehalı der Verbindung nicht zu gering ist, gelang es uns, den Nachweis beider Elemente neben einander in einfachster Weise zu fuhren. Die nicht zu stark salzsaure Lösung, die mit einem Tropfen Schwefelsäure versetzt ist, wurde in der Kälte vorsichtig mit schwefliger Säure uberschichtet und momentan in ein Wasserbad gesenkt. Nach kurzer Zeit scheiden sich alsdann an der Berubrungsstelle beider Zonen die Elemente neben einander in deutlichen Ringen aus; unten das an der rothen Färbung erkennbare Selen, uberschichtet von einer rascher sich ausbreitenden schwarzen Färbung von Tellur.

Die quantitativen Analysen wurden nach den bekannten Methoden im Allgemeinen in folgender Weise ausgefuhrt. Die sorgfällig von Gangart mechanisch gereinigte fein gepulverte Substanz wurde im Einschmelzrohre bei $160^{\circ}-180^{\circ}$ mit rauchender Salpetersäure oxydirt, die zur völligen Verjagung der Salpetersäure zweimal mil Wasser aufgenommene und wieder abgedampfte weisse Masse in ca. $10 \%$ Salzsüure gelöst, die vorbandene Gangart abfiltrirt und im verdunnten Filtrate mit Chlorbaryum die Schwefelsäure gefältt. Das gebildete Baryumsulfat wurde nach dreistündigem Erhitzen auf dem Wasserbade und zwölfstundigem Stehen abfiltrirt, nach dem Gluhen nocbmals mil heisser, verdünnter Salzsäure ausgewaschen und gewogen. Im Filtrate wurden theilweise die Metalle direct mit Schwefelwasserstoff gefallt und mit gelhem Schwefelammonium getrennt, theils nach der Entfernung des uberschüssigen Chlorbaryums das stark salzsaure und erwärmte

1) Zeitschr. f. anal. Chemie 13, 446.

2) Ebenda 26, 243.

3) Zeitschr. f. anorg. Chemie 12, 118. 
Filtrat nach Zusalz von wenig Chlorkalium mit Schwefeldioxyd gesälligt. Das hierbei zugesetzte Ghlorkalium batte zur Folge, dass das gefällte Tellur nicht so viel Wismuth enthielt, als es sonst oft der Fall ist, eine wichtige Vereinfachung bei der quantitativen Trennung. Die Fällung wurde nach zweitägigem Stehen abfiltrirt, abermals mit Salpetersäure oxydirt, und dann wie oben mit Schwefelwasserstoff und Schwefelammonium die Trennung bewerkstelligt. Das Filtrat, das nur die Hauplmenge des Wismuths enthiel, wurde zur Verjagung der schwefligen Säure eingedampft, aus der schwach salzsauren Lösung das Wismuth als Sulfid gefällt und zur Wägung gebracht. Wir verfuhren theilweise aus $Z$ weckmässigkeitsgründen in der geschilderten Weise, weil die Trennung mit Schwefelammonium bei grossen Wismulhund geringen Tellurmengen so bequemer und rascher ermöglicht wird; denn einerseils wird weniger Schwefelammon benöthigt, andererseits kann nach dem Eindampfen der Sulfotelluritlösung im Oxydationstiegel rascher wieder oxydirt und das Tellur sofort wieder mit schwefliger Säure gefällt werden. Zur vollstandigen Abscheidung des Tellurs wurde dies erst nach zweilagigem Stehen filtrirt und als solches gewogen. Das bei der Behandlung des Schwefelwasserstoffniederschlages mit gelbem Schwefelammonium ungelöst bleibende Wismuthsulfid wurde nach kurzem Auswaschen mit verdünnter Schwefelammonlösung sammt dem Filter nochmals oxydirt und abermals"als Wismutbsulfid’gefältt und zur Wägung gebracht.

Der Tetradymit von Orawicza war in kleine nscbuppenförmigen weisslichgrau glänzenden Blättchen in Kalkspath eingesprengt; ausserdem noch begleitet von wenig Kupferkies und Fahlerz und ziemlich viel unlöslöslicher Gangart (Silicate). Die mechanische Trennung war sehr erschwert, da die Adern reinen Materials nur sehr geringe Dimensionen hatlen. Der hohe Procentgehalt an löslicher und unlöslicher Gangart lässt auch das bedeutend niedere specifische Gewicht erklären; "dasselbe wurde zu 5,8637. bestimmt. Die qualitative Analyse ergab: $\mathrm{S}, \mathrm{Te}, \mathrm{Bi}, \mathrm{Cu}, \mathrm{Fe}, \mathrm{Ca}, \mathrm{CO}_{2}$, und Silicale, jedoch kein $S e$. Zur quantitativen Bestimmung wurde das Mineral erst mit verdünnter Salzsäure gewaschen, dann rasch wieder getrocknet und wie oben erwähnt, weiter verfahren. Die Analysen ergaben nachslehende Resultate.

$$
\begin{aligned}
& \text { I. Angew. Menge : } 0,3009 \mathrm{~g} \\
& \text { Gefunden: Gangart: } 0,0323= \\
& \mathrm{BaSO}_{4} \quad 0,0883=S \quad 0,01271=4,03 \quad 4,51 \% \mathrm{~S} \\
& B i_{2} S_{3} \quad 0,1948=B i 0,1583=52,62 \quad 58,93 \quad B i \\
& \text { Te } \quad 0,0948=\quad 31,52 \quad \frac{35,30 T e}{98,74 \%} \\
& 98,91 \% \overline{98,74 \%}
\end{aligned}
$$


II. Angew. Menge: $0,5498 \mathrm{~g}$

Gefunden: Gangart: $0,0625=$

Nach Abrechnung

$$
\begin{aligned}
& \mathrm{BaSO}_{4} \quad 0,1586=S \quad 0,02178=3,96 \\
& 11,11 \% \text { der Gangart: } \\
& \mathrm{Bi}_{2} \mathrm{~S}_{3} \quad 0,3559=B i \quad 0,28917=52,60 \\
& 4,47 \% \mathrm{~S} \\
& \text { Te } \quad 0,1733=
\end{aligned}
$$

III. Angew. Menge: $0,1.194 \mathrm{~g}$

Gefunden: Gangart: 0,002

Nach Abrechnung der Gangart:

$$
\begin{aligned}
& S \text { nicht bestimmt } \\
& \mathrm{Ji}_{2} \mathrm{~S}_{3} \quad 0,3075=\text { Bi } 0,24985=59,50 \% \quad 59,86 \% \\
& \text { Te } \quad 0,1460=\quad 34,81 \quad 34,97
\end{aligned}
$$

Dividirt man nun die Procente durch die Atomgewichte, so ergiebt sich:

$$
\begin{aligned}
& S: B i \quad T e \\
& \text { für 1. } 0,1 / 11: 0,2833: 0,2779 \\
& \text { - II. } 0,1397: 0,2853: 0,2800 \\
& =2: 2: 2
\end{aligned}
$$

hieraus resultirt die Formel $B i_{2} T e_{2} S$ oder $2 B i_{2} T e_{3} . B i_{2} S_{3}$.

Die theoretische Zusammensetzung verlangt

$$
\begin{array}{lc}
S & 4,59 \% \\
B i & 59,52 \\
T e & 35,89 \\
\cline { 2 - 2 } & 100,00 \%
\end{array}
$$

Bei dem Tetradymit von Schubkau konnte leichter analysenreines Malerial beschaffit werden; es lag in grösseren, stark melallisch glänzenden, zum Theil gut ausgebildeten Krystallen, die deutliche Spaltbarkeit nach der Basis zeigten, vor und war in porösem, thonigem Gestein eingebettet. Die qualitative Prüfung ergab neben wenig Gangart $S, B i$, Te und $S e$; lelzteres jedoch nur in sehr geringer Menge, die deshalb auch bei der quantitativen Untersuchung nicht zur Wägung gebracht werden konnte. Das specifische Gewicht betrug 7,0946. Die Resultate von drei Analysen waren folgende:

I. Angew. Menge: $0,4446 \mathrm{~g}$ Gefunden: Gangart: $0,002=$ $\mathrm{BaSO}_{4} \quad 0,1355=$

$$
B i_{2} S_{3}
$$

$\mathrm{Te}$

Se unwăgbar
Nach Abrechnung $0,45 \%$ der Gangart:

$$
4,20 \% S
$$$$
60,36 \quad B i
$$
35, $25 T$ Te

$$
35,09
$$


II. Angew. Menge: 0,4524 g

Gefunden: Gangarı: $0,002=$

Nach Abrechnung

$\mathrm{BaSO}_{4} 0,1426=S \quad 0,01958=4,32 \quad 4,35 \% \mathrm{~S}$

$B i_{2} S_{3} \quad 0,3325=B i 0,27015=59,72 \quad 59,98 \quad B i$

Te $\quad 0,1592=\quad 35,19 \quad 35,35 \quad T e$

Se unwägbar

III. Angew. Menge : $0,3765 \mathrm{~g}$

Gefunden: Gangart: $0,0026=$

$\overline{99,67 \%} \frac{-}{99,68 \%}$

$$
\begin{aligned}
& \mathrm{BaSO}_{4} \quad 0,1195=S \quad 0,01641=4,36 \\
& \mathrm{Bi}_{2} \mathrm{~S}_{3} \quad 0,2777=B i \quad 0,22563=59,93 \\
& \text { Te } \quad 0,1334= \\
& 35,43 \\
& \text { Se unwägbar } \\
& 4,39 \% S \\
& 60,34 \quad B i \\
& 35,68 \quad T e \\
& \frac{-}{100,41 \%} \frac{\text { Se }}{100,41 \%}
\end{aligned}
$$$$
\text { Nach Abrechnung }
$$

$0,69 \%$ der Gangart:

Die Procentzahlen durch die Alomgewichte dividirt ergeben:

$$
\begin{array}{rccccc} 
& S & : & B i & : & T e \\
\text { für I. } & 0,1314 & : & 0,2902 & : & 0,2775 \\
\text { - II. } & 0,1359 & : & 0,2884 & : & 0,2783 \\
\text { - III. } & 0,1372 & : & 0.2901 & : & 0,2801 \\
- & 1 & : & 2 & : & 2
\end{array}
$$

Auch hieraus resultirt die Formel $B i_{2}(T e, S, S e)_{3}$ oder $2 B i_{2} T e_{3} . B i_{2} S_{3}$; es haben somit die Tetradymite von Oravicza und Schubkau gleiche $\mathrm{Zu}$ sammenselzung.

\section{Grünlingit, ein neues Mineral.}

Das Cumberlànder Tellurmineral wár, wie schon oben erwähnt, bisher nur von R a mmelsberg untersucht worden: nach seinen Resultaten scheint ihm kein reines Material zur Verfugung gestanden zu haben; er fand $S 6,43 \%, B i \cdot 84,33 \%$ und $T e$ 6,73\% und nahm unter Ergänzung der Differenz auf $100 \%$ mil $T e$ die Formel $B i_{6} T e S_{3}$ oder $B i_{2} S_{3} . B i_{4} T e$ an.

Das uns von Herrn Prof. Dr. P. Groth uberlassene Material bestand aus unregelmässig begrenzlen, metallisch glänzenden Massen, welche eine äusserst vollkommene und die ganze Masse einheitlich durchsetzende Spaltbarkeit nach einer Richlung besitzen. Dieselben gehören dem gleichen Krystallsysteme an, wie Telradymit, da nach der im hiesigen Inslitute ausgefuhrten Untersuchung des Jerrn H. S mith aus London die Spaltungsebene drei genau unter $60^{\circ}$ einander schneidende Knicke und eine kreisförmige Wärmeleitungsfigur (Methode von Röntgen) zeigt. Auf frischen Spaltungsblältchen ist die Farbe eine graue, während äusserlich schwarze Anlauffarbe zu beobachten war. Auf den einheitlichen Spaltungsblätchen lässt sich sowohl mil der Lupe als mil dem Mikroskop keine Spur einer 
fremden Einlagerung oder eine Differenz im Aufbau entdecken. Das specifische Gewicht wurde zu 7,321 ermittelt. Die qualitative Prüfung ergab nur $S, B i$ und $T e$. Bei der quantitativen Analyse haben wir jedes einzelne Stückchen gesondert untersucht.

I. Angew. Menge: $0,3432 \mathrm{~g}$

Gefunden: $\mathrm{BaSO}_{4} \quad 0,2326=\mathrm{S} \quad 0,03194=9,31 \% \mathrm{~S}$

$$
\begin{array}{llrl}
\mathrm{Bi}_{2} \mathrm{~S}_{3} & 0,3350=B i 0,2722 & =79,31 & \mathrm{Bi} \\
\mathrm{Te} & 0,0440= & 12,82 \mathrm{Te} \\
\cline { 2 - 3 } & & 101,44 \%
\end{array}
$$

II. Angew. Menge: $0,3948 \mathrm{~g}$

Gefunden: $\mathrm{BaSO}_{4} \quad 0,2702=S \quad 0,03711=9,40 \% \mathrm{~S}$

$$
\begin{array}{llcl}
\mathrm{Bi}_{2} S_{3} & 0,3830=B i 0,3112=78,82 & \mathrm{Bi} \\
\mathrm{Te} & 0,0500= & 12,66 & \mathrm{Te} \\
\hline & & 100,88 \%
\end{array}
$$

Die Procentzablen durch die Atomgewichte dividirt ergeben:

$$
S \quad: \quad B i: T e
$$

fur I. $0,2909: 0,3813: 0,10095$

$$
8,727: 11,439: 3,0285
$$

fur II. $0,29373: 0,37895: 0,09972$

$$
8,8119: 11,3685: 2,9916
$$

oder im einfacbsten Verhältniss

$$
3: 4: 1
$$

Dies verlangt die Formel $B i_{4} S_{3} T e$, resp. $B i(S T e\rangle$, worin Wismuth in der zweiwerthigen Form auftritt; es lässt sich unter Annahme der Dreiwerthigkeit noch folgende Formel aufstellen:

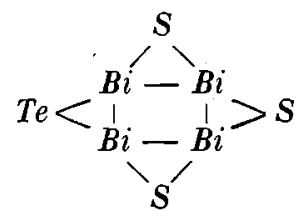

Theoretisch berechnet sich für dieses Verbältniss

$$
\begin{array}{cc}
9,10 \% & S \\
78,86 & B i \\
12,04 & T e \\
\hline 100,00 \% &
\end{array}
$$

Ein Analogon hierzu bietet der in der Natur vorkommende Karelinit $\mathrm{Bi}_{4} \mathrm{O}_{3} \mathrm{~S}$, ein Wismuthoxysulfid, worin Wismuth ebenfalls zweiwerthig auftritt.

Da Herr Dr. F. Grünling, Custos der hiesigen mineralogischen Sammlung, die von uns analysirten Stufen ausgesucht und bestimmt hat, so nannten wir das neue Mineral nach ibm Grunlingit. 